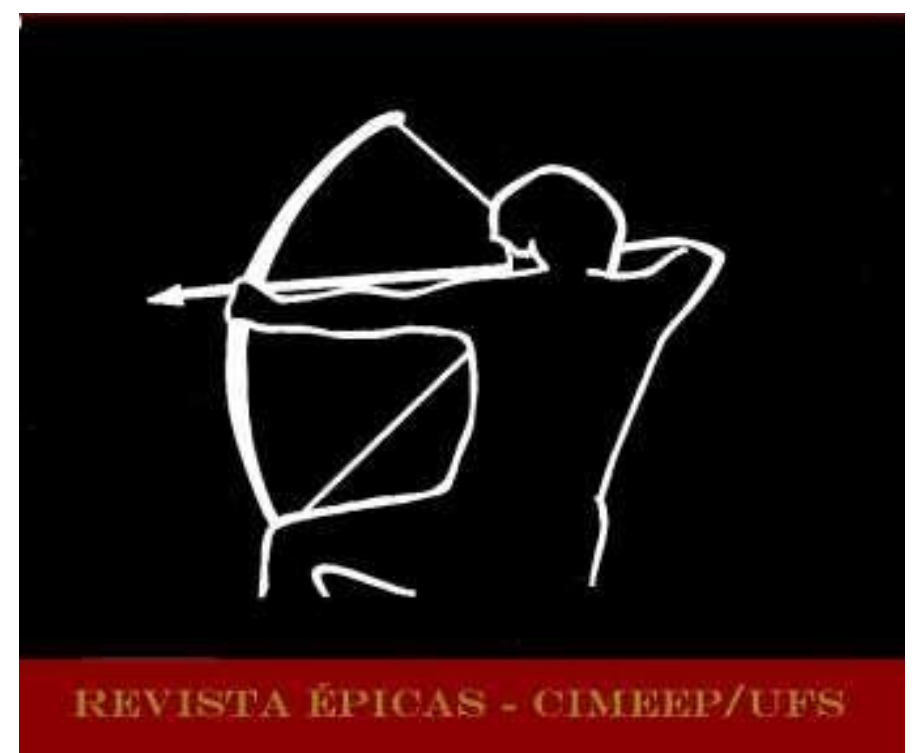

MAUFFREY, Tristan. Les territoires de Gesar: Alexandra David-Néel et la mondialisation de l'épopée tibétaine. In: Revista Épicas. Ano 5, N. 10, Dez 21, p 64-81. ISSN 2527-080-X.

DOI: http://dx.doi.org/10.47044/2527-080X.2021v10.6481

\title{
LES TERRITOIRES DE GESAR : ALEXANDRA DAVID-NEEL ET LA MONDIALISATION DE L'EPOPEE TIBETAINE
}

\section{THE TERRITORIES OF GESAR: ALEXANDRA DAVID-NEEL AND THE GLOBALIZATION OF THE TIBETAN EPIC}

Tristan Mauffrey Université Sorbonne Nouvelle

Résumé: L'épopée de Gesar est étroitement associée, dans les représentations contemporaines, à l'aire culturelle tibétaine, bien que cette tradition poétique soit partagée sous différentes formes par des populations appartenant à des ensembles linguistiques, géographiques et politiques bien plus vastes. Son inscription au Patrimoine culturel immatériel de l'humanité par l'UNESCO en 2009 témoigne des enjeux complexes de la définition d'une géographie épique du Gesar, marquée par un double mouvement de déterritorialisation (par les traductions permettant sa diffusion textuelle en dehors de tout contexte d'énonciation traditionnel) et de reterritorialisation (par la référence explicite, dans ces mêmes textes, à un ancrage local sans cesse redéfini). On se propose ici d'étudier la manière dont la voyageuse et orientaliste Alexandra David-Néel, en élaborant sa traduction-adaptation de 1931 qui fera date dans la réception occidentale du Gesar, joue de ces différents modes de spatialisation du texte épique, pour lui faire reconnaître le statut de chef-d'œuvre de la littérature mondiale.

Mots clés: Gesar, écologie littéraire, politique culturelle, littérature mondiale.

Abstract: The Gesar epic is closely associated, in contemporary representations, with the Tibetan cultural area, although this poetic tradition is shared in different forms by populations belonging to much larger linguistic, geographical and political groups. Its inscription on the UNESCO list of Intangible Cultural Heritage of Humanity in 2009 testifies to the complex issues at stake in the definition of an epic geography of Gesar, marked by a double movement of deterritorialisation (through translations allowing its textual dissemination outside of any traditional context of enunciation) and reterritorialisation (through the explicit reference, in these same texts, to an ever redefined local anchorage). This paper examines the way in which the traveller and orientalist Alexandra David-Néel, in elaborating her 1931 translation-adaptation, which was to become a milestone in the western reception of Gesar, played on these different modes of spatialization of the epic text, in order to have it recognized as a masterpiece of world literature. Keywords: Gesar, literary ecology, cultural politics, world literature. 


\section{Introduction $^{1}$}

Aborder l'Asie par un questionnement sur la notion de géographie épique : pour suivre à notre tour cette voie et contribuer à la réflexion collective, nous nous proposons d'examiner comment s'enchevêtrent différents rapports à l'espace physique, politique et symbolique dans les discours relatifs à la tradition épique de Gesar, à la croisée des études littéraires et de l'ethnographie. Si l'on tente de circonscrire l'objet d'étude " Gesar » comme un ensemble de pratiques narratives et poétiques, parfois musicales, relatives à la tradition légendaire du fameux roi guerrier, une double limitation s'impose d'emblée : cet objet ne peut se réduire à ses formes textuelles écrites, ni évidemment être contenu entièrement dans l'une ou l'autre de ses versions publiées ; mais il ne peut pas non plus être assigné à une aire géographique ou à une communauté culturelle uniques, bref à un territoire déterminé, puisqu'au-delà du Tibet historique cette tradition est partagée sous différentes formes par diverses cultures de Haute Asie et d'Asie centrale, notamment mongolophones. ॥ s'agit donc de questionner ici le geste herméneutique, extérieur à ces cultures traditionnelles, consistant à territorialiser le Gesar, à le définir comme objet littéraire en le situant géographiquement, et ce à plusieurs échelles, pour lui donner une place dans l'espace partagé de la littérature mondiale. Par « mondialisation ", en effet, nous n'entendons pas dans notre titre autre chose que cette opération qui vise à inscrire un ensemble de pratiques énonciatives fortement localisées dans un espace de diffusion et de réception mondialisé.

C'est dans cette perspective que nous aborderons principalement les travaux qu'Alexandra DavidNéel a consacrés à la geste de Gesar à partir des versions écrites et orales qu'elle en a collectées dans le Kham (au Tibet oriental) au début des années 1920. L'adaptation publiée en 1931 sous le titre de La Vie surhumaine de Guésar de Ling, le héros thibétain, racontée par les bardes de son pays a en effet connu un retentissement et une influence durables en rendant la tradition de Gesar accessible au lectorat occidental, atteignant par là-même, selon des modalités que nous discuterons, l'objectif de légitimation explicitement visé ${ }^{2}$. Mais nous commencerons par mettre cette démarche en perspective avec une autre entreprise de mondialisation du Gesar, beaucoup plus récente celle-ci, en analysant la manière dont l'épopée est constituée en "élément culturel » inscrit au Patrimoine immatériel de l'humanité, à l'instigation des autorités chinoises, en 2009. Ces discours s'inscrivent certes dans des contextes bien différents, mais ils nous semblent emblématiques d'un certain mode de construction d'une géographie épique, en ce qu'ils visent à

\footnotetext{
${ }^{1}$ Cet article est partiellement issu de deux communications différentes, l'une dans le cadre du séminaire du groupe de recherche "Antiquité, territoire des écarts" (ATE), et l'autre dans celui du Centre d'études et de recherches comparatistes (CERC) de I'Université Sorbonne Nouvelle. Je remercie toutes les personnes qui m'ont fait des remarques ou des suggestions à l'occasion de ces communications, et tout particulièrement Claudine Le Blanc qui m'a aussi proposé de participer à ce numéro de la Revista Épicas. ${ }^{2}$ La Vie surhumaine de Guésar de Ling est publiée à Paris en 1931 mais l'autrice en supervise immédiatement la traduction anglaise par Violet Sydney, qui paraîtra à Londres en 1933. L'autre publication d'Alexandra David-Néel sur laquelle nous nous pencherons est son article "L'Iliade thibétaine et ses bardes » publié dans le Mercure de France dès septembre 1923, alors que la voyageuse est encore au Tibet. Rappelons que les premières traductions en langues européennes de versions de la tradition épique de Gesar sont dues à Isaac Jacob Schmidt en 1836 et 1839 (traduction russe puis allemande d'une version mongole) puis à August Hermann Fran cke en 1905 (traduction anglaise partielle d'une version tibétaine du Bas-Ladakh), auxquelles Alexandra David-Néel se réfère d'ailleurs.
} 
territorialiser la tradition de Gesar à différentes échelles de manière à la définir et à en assurer la diffusion ou la sauvegarde.

Nous chercherons à en saisir les enjeux épistémologiques à la lumière de la théorie des « écologies littéraires » proposée par le comparatiste Alexander Beecroft dans An Ecology of World Literature (Beecroft 2015). En définissant les aires linguistiques et culturelles de production et de diffusion des œuvres littéraires, anciennes et modernes, comme autant d'environnements qui en conditionnent la réception et l'interprétation, Beecroft envisage en effet l'histoire des textes littéraires (et des discours qu'ils suscitent) dans un cadre à la fois spatial et fonctionnel, à l'échelle de « littératures » pensées comme des systèmes qui entretiennent entre eux des « relations écologiques ». Situées dans l'espace et dans le temps, et prises dans des interactions dynamiques solidaires de l'histoire culturelle, ces littératures peuvent être analysées selon six écologies littéraires (literary ecologies) qui sont moins des catégories closes que des modes de lecture des textes : il s'agit des modèles épichorique (epichoric), panchorique (panchoric), cosmopolite (cosmopolitan), vernaculaire (vernacular), national et global, qui impliquent chacun la prédominance d'un schème culturel de production, diffusion et interprétation des textes à une échelle donnée. Nous reviendrons donc sur certaines de ces catégories pour en faire un usage bien spécifique à propos de la tradition épique de Gesar, afin de questionner la manière dont les discours critiques lui assignent des territoires.

\section{Territorialisation et patrimonialisation de la tradition épique de Gesar}

La candidature à l'inscription de "la tradition épique du Gesar » sur la liste représentative du Patrimoine culturel immatériel de l'humanité a été déposée par les autorités chinoises et acceptée par I'UNESCO en 2009. Dans le dossier de candidature, l'ancrage géographique de cette tradition à l'intérieur de l'espace politique de la République populaire de Chine fait l'objet d'une description précise :

Communautés ethniques tibétaine, mongole et tu de sept provinces et régions autonomes de Chine, à savoir les Régions autonomes du Tibet et de Mongolie intérieure, les Province [sic] du Qinghai, du Gansu, du Sichuan et du Yunnan et la Région autonome ouïgoure du Xinjiang ${ }^{3}$.

Cette caractérisation officielle, qui renseigne la rubrique du formulaire intitulée « Identification de la ou des communauté(s), du (des) groupe(s) ou, le cas échéant, de l'(des) individu(s) concerné(s) et de leur localisation ", consiste donc à inscrire la tradition de Gesar, à l'intérieur des frontières chinoises, dans des espaces relevant de statuts politiques différents (provinces et « régions autonomes ») où elle est associée à plusieurs "communautés ethniques" (nous revenons plus loin sur ce terme officiel). Mais la rubrique suivante, intitulée "Situation géographique et étendue de l'élément", donne lieu à un complément d'information significatif :

3 Formulaire de candidature $n^{\circ} 00204$, consultable sur le site du Patrimoine culturel immatériel de l'UNESCO. URL: https://ich.unesco.org/fr/RL/la-tradition-epique-du-gesar-00204 [consulté le 06/08/2021]. Le dossier est fourni dans ses deux versions officielles, l'anglaise et la française ; c'est cette dernière que nous citons, mais le document précise que le texte original, du point de vue juridique, est la version anglaise. 
Le présent élément (Gesar) est observé essentiellement sur le Plateau du Qinghai-Tibet, dans l'ouest de la Chine, et dans les régions à peuplement nomade du Plateau de Mongolie chinoise septentrionale, situés entre $30^{\circ}$ et $73^{\circ}$ de longitude est et $27^{\circ}$ et $40^{\circ}$ de latitude nord, soit dans une zone s'étendant sur 2700 kilomètres de long d'est en ouest et 1400 kilomètres de large du nord au sud, représentant une superficie totale d'environ deux millions et demi de kilomètres carrés et comprenant les zones agricoles et pastorales du plateau de Mongolie chinoise septentrionale, le Plateau d'Ali, la vallée du fleuve Yarlung Tsangpo, les prairies du Nord-Tibet, la région montagneuse du Hengduan, les montagnes du Gnyan-chen-thang-la, le cours supérieur du fleuve Yangtze et la source du Fleuve jaune, et le versant nord de l'Himalaya. En dehors des vastes zones dans lesquelles il est présent en Chine même, on constate que le Gesar intéresse également des territoires situés loin au-delà de la frontière des États-nations. Il est en effet présent jusque dans la région barti du Pakistan, au Népal, au Bhoutan, au Ladhak [sic] indien, en Mongolie et chez les Kalmouks et les Bouriates de Russie 4 .

La vaste aire de diffusion culturelle de ce qui est désigné sous le terme de Gesar (il est précisé plus loin que la tradition mongole de Geser est incluse sous cet intitulé unique " pour des raisons de commodité ») est ainsi située dans la géographie physique à la fois chinoise et extra-chinoise, pour prendre en compte son extension dans le monde himalayen, en Haute Asie et en Asie centrale. Le cadre national est à la fois affirmé (puisque la candidature est une demande officielle déposée par la République populaire de Chine auprès d'une instance internationale) et transcendé, ce qui est révélateur de l'interaction que nous voulons souligner entre de multiples niveaux de caractérisation spatiale, politique et culturelle de l'objet Gesar (ou " élément », dans les termes du document). Cette désignation permet d'englober la tradition épique sous ses différentes formes d'énonciation orale (accompagnée ou non de musique) dans des contextes de performance rituelle variés, mais aussi sous ses formes de circulation écrite (textes manuscrits et imprimés), ainsi que toutes les autres manifestations actualisant la figure héroïque de Gesar, que cela soit à travers des pratiques cultuelles, des festivals culturels, ou par l'édification de statues, monuments ou musées, par exemple. Ces traces, auxquelles il faudrait ajouter de nombreux toponymes, inscrivent dans le territoire la présence d'une tradition vivante et multiforme autour du souverain mythique ${ }^{5}$. Mais l'élément est d'abord défini comme "épopée ", et c'est donc sa composante littéraire qui donne à l'objet culturel sa cohérence, selon le point de vue adopté dans le dossier de candidature.

Si nous relisons cette description en recourant aux catégories analytiques d'Alexander Beecroft, on peut en effet considérer que plusieurs perspectives, donc plusieurs " écologies littéraires », se superposent et s'entrecroisent dans cet échantillon du discours officiel chinois. C'est d'abord dans la perspective « nationale », on l'a dit, que s'inscrivent ces définitions : de fait, les représentations contemporaines du système littéraire mondial sont largement tributaires de cette écologie qui en constitue le principal fondement conceptuel pour des raisons historiques. Précisons que la catégorie que Beecroft nomme « écologie littéraire globale » n'est pas opérante ici car elle ne rejoint pas la notion de patrimoine culturel mondial, même si l'inscription d'un objet au PCl suppose qu'il présente une valeur pour l'humanité dans son

${ }^{4}$ Ibid.
${ }^{5}$ Nous reprenons la notion de « trace » employée en ce sens par Rolph A. Stein dans son étude fondamentale de la tradition tibétaine
de Gesar (Stein 1959). Il dresse une liste, impressionnante par l'érudition dont elle témoigne, de 89 lieux géographiques associés à la
mémoire du héros par la présence d'un monument, d'un toponyme, d'une pratique rituelle ou poétique par exemple (p. 109 à 135). 
ensemble. Cette catégorie, dans le modèle théorique de Beecroft, pointe plutôt vers un devenir possible de la littérature, mondialisée dans sa forme comme dans son contenu et sa diffusion, tandis qu'il s'agit dans notre cas de donner à une œuvre inscrite dans des cultures nationales une reconnaissance et une visibilité à l'échelle mondiale par l'intermédiaire d'une instance de légitimation internationale (c'est-à-dire fondée sur la juxtaposition de cultures nationales en interaction mutuelle) comme l'UNESCO. Le cadre conceptuel est donc bien celui de l'écologie nationale.

Mais la catégorie de nation présente, dans le cas chinois, une ambiguïté fondamentale et spécifique que l'on retrouve dans la présentation du Gesar telle qu'elle est développée dans le dossier de candidature. En effet, comme le rappelle l'anthropologue Joël Thoraval, un même terme officiel, celui de minzu 民族, "dénote aujourd'hui, en Chine continentale, des réalités traduites, selon le contexte, par "ethnie", "nationalité" ou "nation" » (Thoraval, 1999, p. 45). Dans son analyse de l'histoire de ce " concept officiel », marquée par un «coup de force linguistique, opéré au début des années 1960 par les autorités communistes » (ibid.), Joël Thoraval souligne les enjeux politiques mais aussi épistémologiques de l'emploi de ce terme :

Au terme de ce processus, la Chine est une minzu, la « nation chinoise » (Zhonghua minzu), mais elle est elle-même composée de 56 minzu (les Han, largement majoritaires, et 55 " nationalités minoritaires » (ou shaoshu minzu). (Thoraval, 1999, p. 47)

C'est ce terme que nous retrouvons derrière les " communautés ethniques » mentionnées par le document soumis à I'UNESCO, et qui permet de faire du Gesar une œuvre appartenant à plusieurs littératures nationales en même temps, au sein des frontières nationales de la Chine et en dehors :

En dehors des communautés tibétaine, mongole et tu, le Gesar est vivant au sein de groupes ethniques voisins aussi divers culturellement que les Yugur, Monba, Lhoba, Nahki, Purmi et Bai vivant en Chine, mais on peut également observer à quel point il est répandu au-delà des frontières des États-nations. Il est présent depuis longtemps entre le Népal, le Bhoutan, l'Inde, le Pakistan, la Mongolie, jusque chez les Kalmouks et les Bouriates de Russie, et il s'est avéré être un rare trésor culturel de la Chine et du $\mathrm{PCl}$ commun à l'humanité tout entière ${ }^{6}$.

Comme le montre cet extrait, l'aire de diffusion du Gesar est définie à l'échelle nationale (aux deux sens du terme : celui de chaque " communauté ethnique » et celui de la République populaire de Chine à laquelle elles appartiennent) et dans son extension transnationale à la fois. En prenant acte du fait que ce territoire déborde les frontières géographiques mais aussi les limites conceptuelles de l'État-nation, le discours officiel chinois ressortit également à la perspective « cosmopolite » au sens de Beecroft, dont il adopte certaines caractéristiques.

En effet, l'écologie littéraire cosmopolite est souvent associée à l'existence d'un "vaste espace transculturel, translinguistique, transpolitique, à l'intérieur duquel une unique langue littéraire est prédominante ${ }^{7}$ »; c'est dans ce sens que l'indianiste Sheldon Pollock, auquel Beecroft emprunte le concept,

\footnotetext{
${ }^{6}$ Formulaire de candidature à l'inscription du Gesar au $\mathrm{PCl}$, op. cit.

${ }^{7}$ Beecroft 2015, p. 105 : « a literary cosmopolis, a vast, transcultural, translingual, transpolitical space within which a single literary language predominates ». Nous traduisons.
} 
a décrit comme une cosmopolis la vaste partie de l'Asie du Sud et du Sud-Est dans laquelle, entre le IV et le XIV ${ }^{e}$ siècle, le sanskrit a été pratiqué comme langue de prestige associée à des rapports de domination politique, culturelle et symbolique ${ }^{8}$. Mais Beecroft insiste sur le fait que les catégories qu'il propose sont moins des cadres limitatifs que des modes de lecture, des perspectives adoptées sur les traditions textuelles et leurs usages.

Le caractère plurilingue de la tradition de Gesar, répartie notamment entre les aires tibétophone et mongolophone, ainsi que la prédominance de sa circulation orale en contexte traditionnel pourraient donc apparaître comme des obstacles à l'emploi de cette catégorie critique, en l'absence d'une " unique langue littéraire $»^{9}$. Mais par d'autres aspects, elle peut s'avérer pertinente pour désigner le processus par lequel l'État chinois, se présentant comme le dépositaire privilégié (par la présence de ses « minorités » et par son action scientifique et politique) d'un patrimoine culturel transnational, s'affirme comme puissance régionale et internationale sur le plan culturel. L'un des aspects de cette démarche est le chantier de compilation et d'édition d'un corpus de référence pour la tradition épique tibétaine de Gesar, susceptible de constituer un texte canonique, standard et fixé dans sa forme ${ }^{10}$. Bien que le principe même d'établir une version textuelle complète soit incompatible avec la réalité d'une pratique poétique vivante et mouvante, ce processus de fixation va de pair avec le phénomène de patrimonialisation de la tradition de Gesar au sens large (incluant spectacles, adaptations audiovisuelles, représentations figurées, musées ou expositions, et toutes sortes de célébrations festives), tel que l'a notamment étudié l'ethnomusicologue Nathalie Gauthard. Celle-ci interroge le « processus d'appropriation culturelle » qui accompagne l'évolution récente du spectacle vivant dans les régions de culture tibétaine autour de la figure de Gesar, avec le risque de " muséification » et de « fossilisation » que comporte cette démarche de sauvegarde, très encadrée, d'un héritage traditionnel ${ }^{11}$.

Dans l'étude qu'elle a consacrée à l'histoire de la réception chinoise de la « Gesariade tibétaine » et aux différentes formes d'appropriation symbolique dont celle-ci fait l'objet, sous le titre "Gesar de Pékin ? Le sort du Roi Gesar de Gling, héros épique tibétain, en Chine (post-)maoïste ", Lara Maconi montre également que la figure héroïque de Gesar constitue, aux yeux des autorités chinoises, non seulement un modèle littéraire et artistique mais également un modèle éthique de bon patriote, à promouvoir à la fois auprès des populations de culture tibétaine et à un niveau " pan-chinois ", c'est-à-dire national (Maconi 2004, p. 400-404).

La patrimonialisation du Gesar est ainsi concrétisée à plusieurs échelles (inscription sur les listes du patrimoine culturel immatériel national en 2006, provincial et régional en 2007, et donc mondial en 2009), ce qui recoupe la volonté du pouvoir chinois de s'affirmer comme garant et défenseur actif, sur le plan

\footnotetext{
8 Voir Pollock 2007.

${ }^{9}$ Il faut cependant noter que le prestige religieux et culturel de la langue tibétaine classique a perduré pendant des siècles en Asie

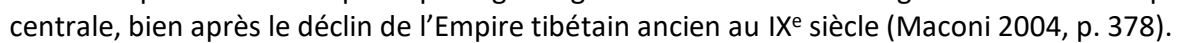

10 Lara Maconi (2004, p. 372), citant le tibétologue chinois Yang Enhong, parle d'un corpus de 120 volumes édités en Chine à la fin des années 1990 .

11 Elle conclut : "La reconnaissance par l'UNESCO de Gesar justifie et renforce une politique envers les minorités qui se veut "primitiviste", paternaliste et protectionniste » (Gauthard 2011, p. 187).
} 
politique, d'un patrimoine inscrit dans plusieurs espaces géographiques à la fois. C'est pourquoi le discours officiel chinois peut être analysé comme relevant en même temps d'une perspective cosmopolite et d'une perspective nationale au sens de Beecroft. Nous retrouvons l'articulation de ces différents niveaux de spatialisation dans cet autre exemple :

[L]a grande influence et la puissance expressive de l'épopée Gesar tiennent au contact crucial de traditions localisées, ce qui semble se prêter idéalement à une communication interculturelle plus active entre des communautés et des groupes différents, tant en Chine qu'à l'extérieur ${ }^{12}$. L'inscription aiderait à jeter un pont entre diverses cultures, faciliterait leur compréhension mutuelle et encouragerait le dialogue entre elles, et par là-même une réflexion sur la créativité culturelle mondiale, au service du principe du patrimoine commun de l'humanité.

Derrière le caractère consensuel des formules choisies, la rhétorique déployée ici s'inscrit pleinement dans une perspective cosmopolite en ce qu'elle vise à affirmer le leadership culturel de la Chine sur le plan régional, en même temps que son statut de défenseur du patrimoine sur la scène mondiale. De fait, le mode de lecture cosmopolite, tel que défini par Beecroft, est fréquemment associé à l'impérialisme politique et culturel.

Enfin, les autorités chinoises sont présentées, dans ce même dossier de candidature, comme détentrices d'une responsabilité éducative à l'égard des populations minoritaires ou "communautés locales » dans la découverte et la valorisation de " leur patrimoine culturel commun ». Ce statut est motivé par le rappel de la riche activité scientifique consacrée de longue date, en Chine, à la tradition de Gesar :

Depuis les années 50, la Chine n'épargne aucun effort pour étudier, recueillir, compiler et sauvegarder la tradition épique Gesar. [...] La proposition d'inscription [de la tradition de Gesar au Patrimoine culturel immatériel] aidera à susciter une prise de conscience, en particulier chez les jeunes générations des 56 groupes ethniques, de l'importance du patrimoine orale [sic] et de la nécessité de le sauvegarder. De plus, il s'agit également d'un effort crucial pour parvenir réellement à renforcer le sentiment de confiance dans leur propre culture des simples citoyens au sein des minorités ethniques concernées, de rapprocher les membres des différents groupes culturels et d'encourager un dialogue sur un pied d'égalité entre les différentes civilisations.

Comme le rappelle Lara Maconi, la recherche chinoise sur le Gesar est en fait antérieure à la fondation de la RPC (elle remonte aux années 1930) et n'a pas connu un développement continu (elle est en particulier interrompue pendant la période de la Révolution culturelle de 1966 à 1978). Mais l'enjeu est ici de souligner le rôle du pouvoir central dans la collecte et l'étude de « traditions localisées » qui, grâce à cette politique de sauvegarde et de promotion, se verraient attribuer une juste place dans le système national de coexistence entre "différents groupes culturels » et dans le système mondial de dialogue entre "les différentes civilisations ». Cette rhétorique peut être relue à travers la catégorie de l'écologie littéraire " panchorique » forgée par Beecroft.

\footnotetext{
12 Le texte anglais est plus explicite sur cet aspect : " the great influence and expressive power of the Gesar epic lies at a crucial juncture between localized traditions, which seems ideally suited to boost intercultural discourse between different communities and groups, both in and out of China ». Formulaire de candidature à l'inscription du Gesar au PCl, op. cit.
} 
Celle-ci est le complément logique de la catégorie d" "épichorique " que Beecroft emprunte à I'helléniste Gregory Nagy pour désigner une pratique rituelle, culturelle ou poétique destinée à un cadre de performance et de signification local (dans le cas de la Grèce ancienne, à l'échelle d'une polis ou d'un ensemble de cités-États partageant par exemple un même dialecte), par opposition aux pratiques culturelles d'importance panhellénique qui concernent potentiellement l'ensemble du monde grec (comme la participation aux jeux olympiques ou le partage des poèmes homériques). C'est à partir du terme de panhellénique que Beecroft élabore celui de panchorique pour en faire un concept transposable à d'autres aires culturelles : une écologie littéraire de type panchorique se définit comme un ensemble formé par la juxtaposition de diverses cultures locales, et s'appuie généralement sur des corpus canoniques exhibant cet ancrage local des traditions compilées ${ }^{13}$.

Dans le cas qui nous occupe, on peut considérer que bien des approches modernes et savantes de la tradition de Gesar relèvent d'une perspective panchorique : la démarche ethnographique qui consiste à collecter des versions écrites et surtout orales, nécessairement localisées par leur forme (linguistique, poétique, performée) et par leur signification pragmatique, pour les intégrer dans un ensemble présupposé cohérent (l'épopée de Gesar comme grand récit et comme objet culturel partagé par des peuples divers) consiste bien à lire chaque version d'un épisode du Gesar comme l'actualisation locale et particulière d'une tradition narrative commune et unitaire, qui n'est pourtant qu'une construction intellectuelle. Cette démarche, que l'on retrouve notamment dans le discours officiel chinois mais aussi dans l'œuvre d'Alexandra David-Néel (bien qu'elle relève alors d'une stratégie tout à fait différente), postule donc, au moins en théorie, un récit canonique du Gesar, en fonction duquel est considérée chaque version locale, partielle et particulière. II n'est évidemment pas question pour nous de minimiser l'importance de l'ancrage local de chaque version connue, puisque les énonciations traditionnelles de ces versions s'inscrivent dans un hic et nunc; pas question non plus de nier la nécessité pratique de désigner comme un tout notionnel la somme de ces versions. Mais nous remarquons simplement que le caractère "panchorique " des approches non traditionnelles du Gesar consiste à articuler deux types de territorialisation (un ancrage local et un partage transculturel), et donc deux types de textualisation (d'un côté, des énoncés toujours spécifiques, et de l'autre, un artefact inexistant qui serait le récit canonique du Gesar). Ce qui est en jeu dans ces processus de spatialisation, c'est bien la possibilité de conférer à la tradition de Gesar, comme ensemble discret d'objets distincts (textes, performances, pratiques...), le statut d'une œuvre, intégrée à la littérature mondiale ou au Patrimoine culturel immatériel de l'humanité.

Nous avons vu que la géographie épique de la tradition de Gesar, du point de vue de la politique culturelle chinoise, pouvait être interprétée comme une combinaison originale des perspectives nationale, cosmopolite et panchorique selon la terminologie de Beecroft, et que cette rhétorique spatiale permettait

\footnotetext{
${ }^{13}$ C'est le cas notamment des épinicies de Pindare dans la Grèce ancienne, ou de la section des « Airs des principautés » (Guofeng) du Shijing en Chine, pour ne citer que deux exemples mentionnés par Beecroft.
} 
de légitimer le principe de la création de « réserves d'écosystèmes culturels pour sauvegarder la tradition épique Gesar ${ }^{14}$, tout en donnant à la politique officielle de la Chine un retentissement international. Mais un tel discours est aussi à replacer dans le contexte géopolitique d'une rivalité pour la revendication de cet héritage. En effet, la catégorie d'écologie littéraire nationale est également mobilisée pour relier étroitement la tradition de Gesar, dans sa réception internationale (et notamment occidentale), à l'identité culturelle et politique tibétaine. Pour ne citer qu'un exemple, l'association de cette idée avec le terme de " national » est explicite dans la définition que donne David Shapiro de l'épopée de Gesar de Ling en introduction de sa traduction anglaise: il s'agit de "l'épopée orale nationale du pays du Tibet, comparable à l'lliade et à |'Odyssée $»^{15}$. Le vocabulaire employé et la comparaison récurrente avec les poèmes homériques (sur laquelle nous reviendrons) soulignent ici la revendication politique d'un état tibétain indépendant dotée $d^{\prime}$ une œuvre fondatrice nationale ${ }^{16}$.

Mais la qualification d'épopée nationale se trouve déjà dans la présentation qu'en fait Alexandra David-Néel, dans la période de l'Entre-deux-guerres, avec des enjeux idéologiques bien différents ${ }^{17}$. Voyons donc à présent en quoi les catégories d'analyse empruntées à Beecroft peuvent guider notre lecture des travaux d'Alexandra David-Néel menés sur le terrain au début des années 1920 et aboutissant à la publication en 1931 de son adaptation textuelle de l'épopée tibétaine, qui fera date dans l'histoire de sa réception occidentale. Pour appréhender cet objet, la voyageuse et orientaliste française croise en effet différentes échelles de représentation qui constituent une véritable géographie épique du Gesar.

\section{Alexandra David-Néel sur le terrain}

Quand elle publie La Vie surhumaine de Guésar de Ling, le héros tibétain, racontée par les bardes de son pays, Alexandra David-Néel fait précéder son récit romancé d'une ample introduction dans laquelle elle décrit en détail les circonstances de sa « rencontre avec Guésar » et la manière dont elle a procédé pour recueillir les versions orales et écrites qui lui serviraient de base, après son retour en France en 1925, pour élaborer son propre texte. Cette introduction éclairante pour comprendre l’objet littéraire que constitue

\footnotetext{
${ }^{14}$ Formulaire de candidature à l'inscription du Gesar au PCl, op. cit.

15 Introduction de The Epic of Gesar of Ling dans Kornman, Khandro et Chönam 2012, p. XV : « The Gesar of Ling epic is the national oral epic for the country of Tibet, equivalent to the lliad and the Odyssey ".

${ }^{16}$ Dans l'avant-propos du même ouvrage, la figure tutélaire du Dalaï-lama est également convoquée en tant que chef politique et religieux en exil et que représentant de la communauté tibétaine dans sa globalité, mais celui-ci se montre plus prudent dans sa définition en choisissant le terme de "peuple " et non de nation: "Many ancient peoples have recited epics as a source of inspiration, the renowned Mahabharata and Ramayana in India, the Hamzanama in Persia, the Iliad and Odyssey in Greece, for example. Tales of heroic feats of Gesar of Ling enjoy a similar status among the people of Tibet. » (ibid., p. VII). Voir aussi le titre éloquent d'un article du tibétologue Samten G. Karmay: "Gesar: The Epic Tradition of the Tibetan People » (Karmay 1998), ou encore la place de l'épopée dans l'analyse du nationalisme tibétain et de la notion de tibétanité proposée par Georges Dreyfus (Dreyfus 1998).

17 Selon ses propres mots, il s'agit de «l'épopée de Guésar de Ling, le poème national des Thibétains, dont j'offre, ici, une traduction condensée aux lecteurs français " (p. XI = 28) et plus loin de "l'épopée nationale du "Pays des Neiges" » (p. XV = 31). Nous citons systématiquement, dans cet article, l'édition originale de La Vie surhumaine de Guésar de Ling (David-Néel 1931) dont nous conservons l'orthographe (en particulier la graphie « Thibet », usuelle à l'époque et abandonnée ultérieurement par Alexandra DavidNéel au profit de « Tibet ») et la pagination (en chiffres romains pour l'introduction) mais dont nous indiquons également la référence équivalente dans la réédition de 1992.
} 
cette adaptation, conservée dans les rééditions ultérieures de l'ouvrage, est emblématique de l'entrecroisement de différents modes d'écriture pratiqués par l'exploratrice, qui sont aussi différentes facettes de son œuvre. Le texte est d'abord une présentation des connaissances de l'époque sur la tradition de Gesar et un exposé méthodologique qui explicite le travail de collecte ethnographique auquel se livre l'orientaliste, doublé d'une exploitation philologique des sources écrites (les manuscrits sont déchiffrés parfois avec difficulté par le lama Yongden, fils adoptif et assistant de l'autrice, qui cosigne la publication), bien que cette collecte ne soit pas faite de manière systématique. Mais c'est aussi un récit vivant à la première personne relatant les étapes successives qui ont mené la voyageuse à mettre ses pas dans ceux du héros tibétain (d'où la métaphore de la rencontre), alors que cette étude ne constituait pas un objectif en soi dans les pérégrinations d'Alexandra David-Néel, avant tout désireuse de pénétrer au Tibet interdit. Les caractéristiques de l'écriture du voyage, enfin, se retrouvent évidemment dans cette introduction puisque c'est le parcours physique de l'autrice dans la région du Tibet oriental, avec l'évocation précise des lieux traversés, qui rend possible et légitime la rédaction ultérieure de cette version de l'épopée à partir des notes prises sur place. Cet ancrage géographique du récit dans le pays de Kham (principalement situé dans l'actuelle province chinoise du Sichuan) effectivement parcouru par la voyageuse est le principal effet de ce paratexte exceptionnel, qui ouvre ainsi l'espace épique. On peut parler d'un processus de reterritorialisation.

Ce dispositif est notable dans les trois étapes qui structurent la deuxième partie de l'introduction, la plus narrative, dont le début est explicitement souligné par le sous-titre « Comment j'ai fait connaissance avec Guésar " (David-Néel 1931, p. XXXVII = 46). Dans le récit qu'en fait Alexandra David-Néel, cette rencontre a lieu en trois temps, chacune de ces étapes s'inscrivant dans un espace précis. La première a lieu à Pékin, dans « un quartier reculé » de la capitale chinoise. Il s'agit d'un face-à-face avec une statue de Gesar, dans un temple où est pratiqué le bouddhisme tibétain. Cette " rencontre » donne lieu à deux discussions, l'une avec une dame originaire du Kham, qui évoque auprès de la voyageuse (conformément à certaines traditions millénaristes qui entourent le héros) le jour où celui qu'elle considère comme son compatriote reviendra et où « ses armées parcourront le Thibet, la Chine et le pays des Étrangers » (p. XL = 48), et l'autre avec un moine d'origine mongole revendiquant lui aussi l'héritage local de Gesar : « Je ne sais pas où est Ling, mais Guésar est certainement un Mongol et c'est de la Mongolie qu'il reviendra avec son armée », prophétise celui-ci, avant d'annoncer qu'il « entraînera les millions d'Asiatiques aujourd'hui assoupis, à la conquête du monde » ( $p . X L I=48-49)$. Dans notre perspective, cette première anecdote située à Pékin, donc en dehors de l'aire de diffusion traditionnelle du Gesar, illustre le phénomène de revendications nationales multiples assignant au héros une fonction symbolique de chef de guerre et promettant son retour victorieux suivi d'une expansion conquérante à travers la Chine et le monde entier à partir d'un centre qui est, selon les cas, le Tibet ou la Mongolie. On constate aussi que le contexte des années 1920 permet d'en faire en quelque sorte un héros postcolonial, susceptible de porter la révolte du continent asiatique dans son ensemble contre 
I'impérialisme occidental ${ }^{18}$. La statue déterritorialisée du temple pékinois est donc le point de départ, pour la voyageuse, de la révélation de ce caractère paradoxal de la territorialisation de Gesar : revendiqué comme local par les habitants de différents territoires, et objet possible d'une vénération en dehors des frontières de cette aire culturelle, il est symboliquement "mondialisable " au sens très particulier où un discours millénariste traditionnel peut lui assigner une destinée mondiale.

La deuxième " rencontre » est située, plusieurs années après, au pays de Kham, où Alexandra DavidNéel sur la route de Jakyendo ${ }^{19}$ se trouve invitée chez « le roi de Ling ». Il s'agit d'un potentat local présenté à la grande surprise de la voyageuse comme « le descendant de Guésar de Ling » (p. XLIV-XLV = 51), ou plus précisément comme le descendant du fils adoptif du héros; il lui montre d'ailleurs des copies manuscrites du texte épique qui sont en sa possession. Le paysage traversé ainsi que le château visité font l'objet d'une description qui inscrit dans l'espace réel l'événement amusant d'une rencontre par procuration avec la figure mythique. Mais l'effet est accentué, dans l'édition originale de La Vie surhumaine de Guésar de Ling, par I'insertion de photographies de l'autrice qui donnent à voir ce fameux roi de Ling ainsi que le territoire auquel est associée la mémoire de son illustre ascendant supposé : I'une, représentant la bâtisse sur la colline, est légendée "Le château du roi de Ling au pays de Kham (Thibet oriental) " et l'autre, représentant le campement d'une famille nomade, est simplement sous-titrée « Au pays de Guésar ». Les photographies complètent donc le texte dans un dispositif où la voyageuse, relayant auprès de son lectorat le discours traditionnel local dont elle est la réceptrice, joue à mettre en scène cet affranchissement des limites spatiales et temporelles : le Gesar mythique est relié à un ici et maintenant par le biais de ce rapport généalogique supposé avec les individus rencontrés par Alexandra David-Néel, qui va de pair avec un ancrage géographique précis : elle est sur ses terres ${ }^{20}$.

Enfin le troisième épisode, le plus décisif, a lieu dans la ville de Jakyendo où Alexandra David-Néel séjourne de septembre 1921 à juillet 1922 : la voyageuse se retrouve littéralement nez-à-nez avec un « barde » spécialiste de la tradition de Gesar qui, sous l'effet de crises de possession, s'identifie à Diktchén

${ }^{18}$ Sur la " géographie » de ce retour attendu de Gesar dans les traditions millénaristes (parfois à partir du royaume mythique de Shambhala), Alexandra David-Néel complète son analyse : « Chez tous ceux avec qui j'ai pu m'entretenir de son retour, j'ai constaté un même espoir: Guésar relèvera la force de l'Asie humiliée, il en chassera les Blancs. Et, détail troublant, parmi ceux qui l'exprimaient avec ferveur, la plupart manquaient totalement de notions géographiques concernant l'Asie et n'avaient jamais vu de Blancs. L'Asie - terme qu'ils n'emploient point - représentait pour ces Thibétains, d'abord leur pays : “le Pays de la Religion”, puis la Chine, l'Inde, le Népaul, la Mongolie et, tout au nord, la mystérieuse terre des “Orossos" (les Russes de la Sibérie). Les Blancs étaient pour eux ces redoutables et pervers Pilings qui commandent dans l'Inde, se sont imposés en Chine et ont convaincu le Dalaï Lama d'exiger de ses sujets des impôts inconnus autrefois » (p. LVII = 59).

${ }^{19} \mathrm{C}^{\prime}$ est-à-dire Jyekundo, aujourd'hui Gyêgu ou Jiegu, dans la province chinoise du Qinghai.

20 L'association de Gesar au royaume de Ling (ou Gling), dans le Kham, ne va cependant pas de soi : comme le rappelle Lara Maconi après Rolf Stein, cette localisation est due à une appropriation de la mémoire du héros par les souverains de cette région, peut-être dès le XVII e siècle (voir Maconi 2004, p. 374-375 et Stein 1959, p. 572). Mais bien d'autres interprétations de ce nom sont possibles, comme l'explique également Georges Dreyfus : "Le pays de Ling correspond, pour une large part, au Dzambouling, le continent méridional de la cosmologie bouddhique. La légende ne concerne donc pas seulement la fondation d'une principauté obscure du Tibet oriental, mais aussi la création du monde des humains : Ling est le monde des hommes sauvés par le héros, envoyé par les dieux. II ne représente pas simplement Lingtshang ou le Tibet oriental : il est plutôt un symbole à dimensions multiples qui représente diverses réalités, selon le temps et le lieu. Pour certaines populations du Tibet oriental, au xIX siècle, Ling était en effet leur propre pays, victime des empiètements du gouvernement central. En d'autres régions du Tibet, ou en d'autres temps, Ling est le Tibet luimême, le pays construit par les hauts faits des rois vertueux " (Dreyfus 1998, p. 43-44). 
Chémpa (un ministre du roi mythique) dont il serait la réincarnation. C'est cette rencontre fortuite qui va se prolonger par des séances de performance poétique, à la demande d'Alexandra David-Néel et de Yongden, au cours desquelles ils prendront des notes sur cette version locale de l'épopée. Elle est donc d'une importance capitale dans le processus qui mène à l'édition du texte que le lecteur français a sous les yeux. Mais c'est aussi l'aboutissement de ce cycle de trois « rencontres » avec Gesar : après le face-à-face avec la statue du héros puis avec son prétendu descendant, il s'agit ici d'une rencontre avec l'épopée elle-même, puisque Gesar est contenu dans la tradition poétique qui le fait exister. La situation d'énonciation est construite artificiellement: Alexandra David-Néel demande au "barde », ou sgrung-mkhan en tibétain, d'exécuter pour elle et son fils adoptif une série de performances qui va durer " plus de six semaines ». Mais celle-ci est précédée par une séance "normale » de performance poétique en contexte traditionnel, à laquelle les voyageurs assistent dès le lendemain de la rencontre. La sensibilité ethnographique d'Alexandra David-Néel trouve ici ses limites : elle rapporte des éléments essentiels de ce mode d'énonciation spécifique (comme les interventions de l'auditoire par des exclamations rituelles, ou la valeur rythmique de formules poétiques récurrentes) mais elle ne sait pas les interpréter comme appartenant pleinement à la performance épique, dont elle veut avant tout suivre la logique narrative ${ }^{21}$. C'est en tout cas pour la voyageuse une immersion dans un contexte où le héros appartient à la mémoire culturelle partagée, activée par la pratique poétique.

Sur le terrain, donc, Alexandra David-Néel côtoie en quelque sorte les protagonistes de la tradition de Gesar, fait l'expérience de sa présence dans l'imaginaire et dans la culture vivante des Tibétains, et collecte les traces orales et écrites de ses énonciations poétiques. Les photographies prises in situ par l'autrice, c'està-dire sur les lieux mêmes de sa réception qui sont aussi des lieux auxquels réfère le récit (du moins dans cette version), complètent le dispositif textuel dans l'édition de 1931 : celui-ci vise à traduire et transposer dans la culture du lecteur un mode traditionnel d'actualisation de la figure de Gesar, selon lequel un énoncé fortement localisé est susceptible d'abolir la distance temporelle qui sépare la matière de l'épopée et ses destinataires (par exemple la performance d'un barde, adaptée au contexte spatial de son énonciation, fait exister rituellement ici et maintenant la tradition mythique de Gesar). Tout le paratexte sert à construire un équivalent fonctionnel de cette pragmatique traditionnelle, puisqu'Alexandra David-Néel récrit une version de l'épopée en lui ôtant toutes les caractéristiques de sa poétique propre, et qu'elle recrée pour les lecteurs un contexte d'énonciation fictif autour d'un texte qui n'a jamais été énoncé comme tel. C'est pourquoi l'introduction et les photographies lui permettent de s'inscrire, avec son compagnon de voyage et d'écriture, dans cet espace qu'ils ont parcouru physiquement et qu'ils font parcourir mentalement aux lecteurs. En

\footnotetext{
${ }^{21}$ Les enjeux ethnopoétiques de cette description de la performance débordent le cadre de cet article. Pour une analyse éclairan te de tous les éléments de l'énonciation poétique traditionnelle relevés par Alexandra David-Néel sans être interprétés, voir l'étude que leur consacre l'ethnomusicologue Mireille Helffer (Helffer, 1977). Je remercie Nadine Gomez et Marion Dapsance pour leur aide dans ma recherche, au sein des archives de la Maison Alexandra David-Neel à Digne-les-Bains, de documents témoignant de la manière dont Alexandra David-Néel et le lama Yongden ont noté, dans le temps de la performance, les informations relatives à la pratique du barde. Ces recherches n'ont cependant pas abouti, les manuscrits originaux ayant vraisemblablement été détruits par l'autrice après la rédaction de La Vie surhumaine de Guésar de Ling.
} 
témoignent par exemple, outre les photographies déjà citées, la légende du cliché d'un « Pasteur du pays de Guésar montant un yak », au-dessus de celui du lama Yongden (David-Néel 1931, feuille d'illustrations rectoverso non paginée, intercalée entre les p. 126 et 127), ou encore le célèbre portrait de l'autrice posant au milieu de guerriers tibétains : «Au pays des ennemis de Guésar. Mme David-Neel avec des chefs du pays de Hor » (ibid., feuille intercalée entre les p. 78 et 79).

Cet ancrage territorial du Gesar d'Alexandra David-Néel est donc essentiel pour légitimer l'objet textuel et le rendre lisible ; c'est aussi un aspect déterminant de la démarche de l'orientaliste française et de son assistant de culture tibétaine, qui veulent faire reconnaître un statut de texte canonique à l'épopée de Gesar. À la fin de son introduction, l'autrice explique que leur texte (présenté comme une «traduction condensée ", ce qui est pour nous un euphémisme) est le produit d'une réélaboration fondée sur la pratique de la synthèse narrative, le but étant de donner une cohérence et une exhaustivité à la restitution de l’enchaînement des épisodes :

[B]ien qu'élaguée de ses fatigantes redondances, l'épopée de Guésar donnée ici contient toutes les péripéties marquantes des aventures du héros. J'ai fondé mon travail sur les manuscrits que je possède, sur les notes que j'ai prises d'après d'autres manuscrits qui m'ont été communiqués au Thibet et sur celles écrites par le lama Yongden et par moi en suivant les chants de différents bardes.

Bien qu'ils s'accordent dans leurs grandes lignes, manuscrits et bardes ne laissent pas que de diverger sur des points de détail. Lorsque je me suis trouvée en présence de plusieurs récits différents, je me suis attachée à reproduire celui qui paraissait le plus généralement admis. (DavidNéel 1931, p. LXI-LXII = 62)

Dans la perspective d'Alexandra David-Néel, la collecte de versions orales et écrites nécessairement divergentes dans le cas d'une tradition vivante et mouvante doit aboutir à une version standardisée du récit, une sorte de dénominateur commun qui n'a d'autre existence que dans la représentation unitaire de l'épopée que l'autrice a hérité de ses propres catégories littéraires, notamment d'inspiration aristotélicienne. Présupposer, un peu à la manière des éditeurs alexandrins des poèmes homériques, l'existence d'une version originelle et commune donc authentique de l'épopée est une prémisse qui guide la démarche de l'orientaliste : elle justifie à la fois le choix d'une version localisée dans le Kham considéré à l'époque comme le berceau de la tradition de Gesar et de son héros (à cause de la localisation de Gling), et la réélaboration du récit sous une forme standard, comme l'autrice l'annonce dès le début de son introduction :

Si nous considérons que cette version est la plus répandue, qu'elle fait en quelque sorte autorité, non seulement au pays de Kham, tenu pour être la véritable patrie du héros, mais aussi à Lhassa et dans tout le Thibet propre, elle a certains droits au titre de version officielle. Toutefois, il faut se garder de voir en elle l'écho absolument fidèle de la légende primitive. (Ibid., p. XI = 28-29)

La dernière phrase semble restituer à la tradition épique son instabilité et son historicité, mais elle ne fait que confirmer la conception unitaire que l'orientaliste a de l'épopée ; le fait de désigner au singulier « la version » locale du Kham traduit bien son programme: il s'agit de donner de cette tradition poétique multiforme une version canonique, qui serait conforme à ce qu'elle appelle « la version officielle » mais qui reste un artefact textuel. Bien sûr, certains épisodes de la tradition de Gesar sont plus communs, moins mouvants que d'autres dans son aire de diffusion; mais en adoptant ce point de vue centré sur une 
standardisation du récit, Alexandra David-Néel fait du Gesar une lecture cosmopolite au sens où, pour reprendre les catégories d'Alexander Beecroft, elle cherche à dégager une version canonique d'une tradition multiforme. Typique d'une culture littéraire européenne plutôt que tibétaine, cette démarche est indissociable du déplacement qui est opéré par la traduction (en français et en anglais presque simultanément), et qui vise à insérer le Gesar, à partir des catégories littéraires européennes, dans ce que la voyageuse n'appelle pas encore la littérature mondiale.

\section{La migration d'une tradition poétique locale vers la littérature mondiale}

Élaborer, à partir du divers, un récit unitaire et standard est un mode de lecture, extérieur aux pratiques et usages traditionnels du Gesar, qu'Alexandra David-Néel inscrit explicitement dans son expérience de voyageuse. Mais c'est aussi une démarche intellectuelle qui est corrélée à la volonté de contribuer à la diffusion du « patrimoine culturel » (bien qu'elle n'emploie pas ces termes) tibétain auprès $d^{\prime}$ un auditoire occidental. Or cette stratégie s'articule à une pratique bien particulière qui consiste à qualifier la tradition de Gesar au moyen de catégories littéraires européennes, et en particulier de références grecques associées au genre épique. Dès les premières ébauches de son travail sur le terrain, la comparaison avec l'lliade est donc un choix herméneutique que fait Alexandra David-Néel pour saisir cet objet insaisissable qu'est le Gesar et l'insérer dans un cadre conceptuel familier et prestigieux aux yeux de l'orientaliste et de ses lecteurs. Elle emploie l'expression d' " lliade tibétaine », entre guillemets, dans une lettre à son mari du 10 novembre $1922^{22}$, quelques mois après les séances de performance poétique entendues à Jakyendo, pour décrire le travail de collation, de traduction et de comparaison des manuscrits que les deux voyageurs sont en train d'effectuer, et c'est cette expression qui servira de titre accrocheur à l'article publié dans le Mercure de France l'année suivante, première ébauche de la future introduction de La Vie surhumaine de Guésar de Ling, alors qu'ils sont encore au Tibet ${ }^{23}$. Certes, la comparaison sert d'abord à introduire, au moyen de I'analogie, le lectorat dit cultivé dans un monde tibétain qu'il connaît généralement peu : elle est justifiée par l'autrice, dans les premières lignes de l'article, quand elle affirme par un présupposé universalisant qu'il existe chez « chaque peuple " des « chant héroöques glorifiant comme ceux d'Homère les exploits de héros à demi mythologiques dont les origines se perdent dans la nuit des temps " (David-Néel 1923, p. 714). Cette accroche bien superficielle est une manière de gloser le titre de l'article et de poser la comparaison filée par la suite entre l'épopée de Gesar et celle d'Achille; mais c'est aussi un postulat qui sert la stratégie de légitimation de la tradition poétique tibétaine. Par cette analogie, en effet, qui connaîtra une fortune durable ${ }^{24}$, l'article fait de la tradition de Gesar un texte canonique équivalant, par son contenu et par son importance culturelle dans cette partie de l'Asie, aux poèmes homériques, érigés en archétype du genre

\footnotetext{
22 Voir cette lettre dans Alexandra David-Néel, Correspondance avec son mari, édition intégrale 1904-1941, Paris, Plon, 2016, p. 703.

${ }^{23}$ Alexandra David-Néel, « L'Iliade thibétaine et ses bardes » (article publié sous le nom d'Alexandra David dans le Mercure de France du 15 septembre 1923, p. 714-725).

${ }^{24}$ Voir par exemple la mention des poèmes homériques dans l'avant-propos du Dalaï-lama à Kornman, Khandro et Chönam 2012 , cité plus haut.
} 
épique dans l'histoire littéraire européenne. Cette idée sera d'ailleurs reprise dès l'ouverture de la préface rédigée par le grand indianiste Sylvain Lévi pour l'édition de La Vie surhumaine de Guésar de Ling quelques années plus tard : « Le Guésar est l'Iliade de l'Asie Centrale » (David-Néel 1931, p. VII)²5.

De plus, cette comparaison « canonisante " est étroitement associée à la démarche déjà abordée plus haut qui consiste à inscrire l'étude de la tradition rapportée de Gesar dans un espace géographique précis, parcouru par la voyageuse :

Si nous n'avions, pour guider nos recherches concernant la personnalité de Guésar, que le récit de ses hauts faits, nous risquerions d'en savoir aussi peu à son sujet qu'à celui d'Agamemnon, de Ménélas ou du prudent Ulysse.

Un voyage à travers la Grèce moderne ne paraît pas de nature à pouvoir beaucoup nous renseigner sur ces héros ; mais il n'en est pas ainsi au pays natal du Roi Guésar. Que ce dernier ait vécu au VIIII siècle, ainsi que certains le supposent, ou au VIII siècle, comme semblent l'indiquer les chants héroïques thibétains (les Gyal-toung), ou n'importe quand, la différence amenée par le temps est peu sensible dans la contrée où il paraît avoir vu le jour. (David-Néel 1923, p. 714-715)

Dès I'article de 1923, donc, rédigé sur place, Alexandra David-Néel, qui présuppose le caractère historique du héros légendaire, thématise l'expérience unique qu'offrirait le voyage à la rencontre du héros sur les lieux mêmes où sont traditionnellement localisés certains de ses exploits, jugeant que le fait de se consacrer à l'étude d'une culture épique vivante permet d'abolir la distance spatiale et temporelle entre le public occidental moderne et l'objet épique ${ }^{26}$. Cette opération intellectuelle et symbolique ne se limite pas à la prise en compte des circonstances ethnographiques de la collecte du texte poétique : elle vise à constituer la tradition poétique de Gesar présentée dans l'article (bien avant, donc, sa mise en forme romanesque de 1931) en un objet reconnaissable et accessible qu'elle fait migrer jusqu'aux lecteurs français. En effet, c'est paradoxalement la combinaison de ces deux gestes (décrire l'ancrage local de la tradition tibétaine et projeter en même temps sur elle des catégories européennes héritées de la culture grecque) qui rend le Gesar « déterritorialisable », et le met à portée du lectorat européen. De ce point de vue, Alexandra DavidNéel se place dans la lignée d'écrivains voyageurs pionniers du comparatisme au XIXe siècle qui, tel Xavier Marmier en Islande par exemple, déchiffraient sur une terre éloignée les formes vivantes d'une tradition littéraire vénérable à la lumière de leurs références classiques. C'est un tel dispositif, bien plus que les traductions antérieures déjà mentionnées, qui fait alors entrer le Gesar dans ce que nous appelons maintenant la littérature mondiale, au sens de l'ensemble des littératures du monde.

L'aboutissement de ce processus peut être lu dans la préface déjà mentionnée de Sylvain Lévi à l'édition de 1931 : en apportant sa caution académique de premier plan à cette publication (il est alors professeur au Collège de France), le savant parachève le déplacement opéré par la voyageuse, des

\footnotetext{
${ }^{25}$ Notons également que, dans plusieurs références que nous citons ici en respectant la typographie originale, les titres de ces œuvres canoniques ne figurent pas en italique mais en caractères romains, comme si cette présentation allait de pair avec leur statut de monuments plutôt que d'œuvres singulières. Ainsi, " l'lliade » apparaît presque comme une catégorie générique en soi. ${ }^{26}$ On remarque que quelques années plus tard, entre 1933 et 1935, Milman Parry et Albert Lord révolutionneront la compréhension moderne de la poésie homérique en allant écouter sur le terrain, en Serbie et au Monténégro, les chanteurs épiques traditionnels.
} 
montagnes tibétaines vers la bibliothèque universelle (malgré son souhait forcément inassouvi de voir un jour publié « le texte original » de ce Gesar) :

Les rhapsodes le colportent à travers les hauts plateaux où ils mènent leur vie errante. C'est là que Madame A. David-Neel a eu l'occasion de les entendre et de noter leurs récits. En attendant qu'elle puisse nous donner le texte original, remercions-la d'avoir révélé au public français, sous une forme pittoresque et attrayante, une épopée qui pour son importance nationale et sa signification de culture vient se ranger auprès de l'lliade, de l'Énéide, des Niebelungen, du Roland. (David-Néel 1931, p. VIII)

Le simple usage du mot « rhapsode » pour désigner, au présent, l'activité du sgrung-mkhan est significatif à cet égard : dans le parallèle implicitement établi, ces " bardes » sont autant d’Homères tibétains habitant une contrée prétendument figée dans son archaïsme et porteurs d'un héritage patrimonial. Par le processus de déterritorialisation et de reterritorialisation qu'opère l'adaptation d'Alexandra David-Néel, et du fait qu'elle élabore, à partir d'une tradition poétique fortement localisée, un texte littéraire autonome, l'autrice permet au Gesar d'accéder au statut de texte canonique, au côté des chefs-d'œuvre de la littérature mondiale. Mais de quel Gesar s'agit-il vraiment, si celui-ci est défini par des catégories extra-tibétaines ?

\section{Considérations finales}

Nous cherchions à voir comment des discours non traditionnels (le descriptif adressé par les autorités chinoises à I'UNESCO, la présentation par Alexandra David-Néel de son adaptation narrative destinée au lecteur européen curieux, ou encore sa relation des pratiques poétiques observées sur le terrain) élaboraient une géographie épique du Gesar, et en quoi ce geste herméneutique rendait possible la définition même de leur objet en termes étiques, c'est-à-dire extérieurs aux cultures traditionnelles auxquelles appartient le roi mythique. Au terme de ce parcours, revenons sur la notion de « territoire » annoncée dans notre titre. Celleci nous a semblé utile pour questionner la spatialisation d'une tradition épique, à la fois sur le plan de son extension géographique (son aire de diffusion, particulièrement vaste et translinguistique dans le cas du Gesar) et sur le plan de son extension symbolique (le territoire sur lequel le héros règne dans la tradition narrée, auquel il est associé par des toponymes, ou sur lequel il est censé régner un jour, lors de son retour espéré). Articulée aux catégories d'écologies littéraires proposées par Alexander Beecroft, la notion de territoire peut servir à interroger la manière dont ont été interprétés les multiples rapports d'une tradition épique à l'espace, en prêtant attention à chaque fois aux perspectives qui guident ces interprétations (le " cosmopolitisme » à travers lequel nous relisons les écrits d'Alexandra David-Néel de l'Entre-deux-guerres $\mathrm{n}^{\prime}$ a pas le même sens que celui des autorités chinoises au début $d u x \mathrm{Xl}^{\mathrm{e}}$ siècle). Mais le principal enjeu de cette approche critique nous semble être notre réception de traditions culturelles et textuelles distantes : ce qu'elle permet d'interroger, c'est la migration d'une tradition épique comme celle de Gesar, déterritorialisée et donnée à lire à un public qui ne soit pas local, bref virtuellement « mondialisée ». La réflexion critique sur cette réception, et sur la manière dont elle articule les échelles locales, nationales, régionales et mondiales, doit alors aboutir à une autre exigence, qui consiste à intégrer à notre lecture les catégories propres à la 
manière dont circule l'œuvre dans la culture étudiée, en contexte traditionnel ${ }^{27}$. Ce que l'on pourrait appeler une "reterritorialisation critique", cependant, n'est évidemment possible aujourd'hui que parce qu'Alexandra David-Néel, en déterritorialisant après d'autres la tradition de Gesar, a contribué à la faire entrer dans notre culture partagée.

\section{Références bibliographiques}

BEECROFT, Alexander. An Ecology of World Literature: From Antiquity to the Present Day. Londres et New York : Verso, 2015.

DAVID, Alexandra. L'Iliade thibétaine et ses bardes. In : Le Mercure de France, n. 606, 15 septembre 1923, p. 714-725.

DAVID-NEEL, Alexandra et le Lama Yongden. La Vie surhumaine de Guésar de Ling, le héros thibétain, racontée par les bardes de son pays. Préface de Sylvain Lévi. Paris : éditions Adyar, 1931 (rééd. sous le titre La Vie surhumaine de Guésar de Ling. Monaco : éditions du Rocher, 1978, puis Paris : Pocket, 1992).

DAVID-NEEL, Alexandra. Correspondance avec son mari, édition intégrale 1904-1941. Paris : Plon, 2016.

DREYFUS, Georges. Le Nationalisme, entre mémoires glorifiées et identité collective. In : BUFFETRILLE, Katia et RAMBLE, Charles (dir.). Tibétains. 1959-1999 : 40 ans de colonisation. Paris : Autrement, coll. " Monde » HS n. 108, 1998, p. 21-57.

FITZHERBERT, Solomon George. The Tibetan Gesar Epic as Oral Literature. In : DOTSON, Brandon, NORBU GURUNG, Kalsang, HALKIAS, Georgios et MYATT, Tim (dir.). Contemporary Visions in Tibetan Studies. Chicago : Serindia, 2009, p. 171-196.

GAUTARD, Nathalie. L'Épopée tibétaine de Gesar de Ling. Adaptation, patrimonialisation et mondialisation. In : Cahiers d'ethnomusicologie, n. 24, 2011, p. 173-189.

HELFFER, Mireille. Les Chants dans l'épopée tibétaine de Ge-sar d'après le livre de la course de cheval, version chantée de Blo-bzan bstan-'jin. Genève et Paris : librairie Droz, coll. " Hautes études orientales », 1977.

KARMAY, Samten. Gesar : The Epic Tradition of the Tibetan People. In : The Arrow and the Spindle : Studies in History, Myths, Rituals and Beliefs in Tibet, vol. 1. Katmandou : Mandala Book Point, 1998.

KORNMAN, Robin, KHANDRO, Sangye et le Lama CHÖNAM (trad.). The Epic of Gesar of Ling, vol. 1 : " Gesar's Magical Birth, Early Years, and Coronation as King ». Boston et Londres : Shambhala, 2012.

MACONI, Lara. Gesar de Pékin ? Le sort du roi Gesar de Gling, héros épique tibétain, en Chine (post-) maoïste. In : LABARTHE, Judith (dir.). Formes modernes de la poésie épique : nouvelles approches. Bruxelles : P.I.E. Peter Lang, 2004, p. 371-419.

POLLOCK, Sheldon. The Language of the Gods in the World of Men: Sanskrit, Culture, and Power in Premodern India. Delhi: Permanent Black, 2007 (rééd. Berkeley: University of California Press, 2009).

STEIN, Rolf Alfred (éd. et trad.). L'Épopée tibétaine de Gesar dans sa version lamaïque de Ling. Paris : PUF, coll. « Annales du musée Guimet », 1956.

\footnotetext{
27 C'est par exemple ce que fait Solomon George Fitzherbert quand il montre, à partir des catégories poétiques tibétaines, que la tradition épique est parfois décrite comme " faisant partie du paysage » : « In Tibetan idiom the tales are said to "fall" or "cascade" ('bab) through the inspired, entranced or sometimes 'possessed' medium from an extra-mondane source. The transmission of the epic is therefore not just about memorization and oral transmission passed down from generation to generation, but is crucially, and explicitly, in the hands of the spirits. Sometimes the tradition is described as being "part of the landscape" - it is conceived as an intangible organic entity living in the land and in the people " (Fitzherbert, 2009, p. 174).
} 
STEIN, Rolf Alfred. Recherches sur l'épopée et le barde au Tibet. Paris : PUF, coll. « Bibliothèque de l'Institut des Hautes Études Chinoises ", 1959.

THORAVAL, Joël. L'usage de la notion d'« ethnicité » appliquée à l'univers culturel chinois. In : Perspectives chinoises, n. 54, 1999, p. 44-59. 